\title{
The HSP90 Inhibitor, 17-AAG, Influences the Activation and Proliferation of T Lymphocytes via AKT/GSK3 $\beta$ Signaling in MRL/lpr Mice
}

This article was published in the following Dove Press journal:

Drug Design, Development and Therapy

\author{
Liang-Jian Hong \\ $\mathrm{Ai}$-Jun Chen \\ Feng-Zeng Li \\ Ke-Jun Chen \\ Sheng Fang
}

Department of Dermatology, The First Affiliated Hospital of Chongqing Medical University, Chongqing 400016, People's Republic of China
Correspondence: Sheng Fang Department of Dermatology, The First Affiliated Hospital of Chongqing Medical University, I Youyi Road, Yuzhong

District, Chongqing, People's Republic of China

Email fangshengderm@I63.com
Objective: To explore the molecular mechanism of 17-AAG in the treatment of systemic lupus erythematosus (SLE), and the effects of the heat shock protein 90 (HSP90) inhibitor 17-AAG on the activation and proliferation of lymphocytes and the AKT/GSK3 $\beta$ signaling pathway in MRL/lpr mice were detected.

Methods: MRL/lpr mice were randomly divided into the control group and the experimental group. The experimental group was injected intraperitoneally with 17-AAG, and $\mathrm{T}$ lymphocytes were separated by magnetic beads. Lymphocyte proliferation was detected by MTT and flow cytometry (FCM), and the expression of the HSP90 protein and PI3K/AKT signaling pathway-related proteins was detected by Western blotting. Renal histopathology and immune complex deposition were also observed in both groups.

Results: Immune complex deposition and inflammation decreased in kidneys from MRL/lpr mice in the experimental group. HSP90 protein expression, T lymphocyte proliferation and phosphorylated AKT and GSK3 $\beta$ levels also decreased in the experimental group.

Conclusion: 17-AAG can inhibit the activation and proliferation of $\mathrm{T}$ lymphocytes and downregulate the AKT/GSK3 $\beta$ signaling pathway, which may be relevant for the treatment of SLE.

Keywords: 17-AAG, HSP90, AKT, lupus, activation

\section{Introduction}

Heat shock protein 90 (HSP90) is a cytoplasmic molecular chaperone that participates in the maturation and stabilization of a subset of cellular proteins termed "client" proteins. ${ }^{1}$ The number of HSP90 client proteins is very large, and they range in function from cell cycle control to signal transduction and cell growth regulation. ${ }^{2}$ An increasing number of studies have shown that HSP90 plays an important role in autoimmunity and tumor immunity. ${ }^{3,4}$ Although the use of HSP90 inhibitors in the treatment of tumors has been investigated, ${ }^{5,6}$ there have been a few reports about HSP90 inhibitors in the treatment of autoimmune diseases. The etiology and pathogenesis of systemic lupus erythematosus (SLE) have not been fully elucidated thus far. An increasing number of studies have shown that HSP90 plays an important role in autoimmunity and participates in the development of SLE. ${ }^{7,8}$ Studies have reported high levels of HSP90 autoantibodies in SLE patients, and high titers of these antibodies were associated with renal disease and low C3 levels. ${ }^{9}$ Elevated serum levels of HSP90 have been correlated with elevated levels of IL- 6 in SLE patients. ${ }^{10}$ However, there are few studies on the use of HSP90 molecular inhibitors for the treatment of SLE. AKT is 
a downstream effector molecule of PI3K and is thought to mediate many events during biological responses. Therefore, HSP90 molecular inhibitors have become a new potential approach for the treatment of SLE. In this study, the effect of the HSP90 inhibitor 17-AAG on the MRL/lpr lupus mouse model was observed. We also detected the effect of 17-AAG on the activation and proliferation of lymphocytes and the AKT/GSK3 $\beta$ signaling pathway in MRL/lpr lupus mice and explored the mechanism of HSP90 inhibition in a lupus mouse model.

\section{Materials and Methods}

\section{Reagents}

Tanespimycin (17-AAG) (purity: 99.92\%, batch number: s11405) was purchased from Shanghai Selleck Company. The antibodies used for Western blotting were obtained from Cell Signaling Technology (CST, Beverly, MA) and were specific to the following proteins: HSP90 $\beta$, pAKTThr308, pGSK3 $\beta$ Ser9, AKT, GSK3 $\beta$ and $\beta$-actin. The secondary antibody was polyclonal anti-rabbit IgG.

\section{Animals}

Twenty 5-week-old female MRL/lpr lupus mice (weight 18 g-24 g) were purchased from Shanghai Shrek Animal Experiment Co., Ltd. All experimental protocols were performed according to the Guidelines for the Care and Use of Laboratory Animals, were approved by the Institutional Ethics Committee of Chongqing Medical University and conformed to the National Institutes of Health Guide for Care and Use of Laboratory Animals. All animals were kept in the SPF-grade animal laboratory room of the Animal Experimental Center of Chongqing Medical University. MRL/lpr mice were divided into an experimental group and a control group via the random expulsion method. The experimental group was intraperitoneally injected with 17-AAG $50 \mathrm{mg} / \mathrm{kg} 3$ times per week for 7 consecutive weeks from 7 weeks of age. The control group was given the same amount of normal saline.

\section{Renal Histology}

Histological analysis of kidneys from MRL/lpr mice was performed at $14 \mathrm{w}$. MRL/lpr mice were killed by dislocation and soaked with $75 \%$ ethanol for $15 \mathrm{~min}$. The mice were dissected on an ultraclean workbench, and the kidney from one side was quickly collected. The kidneys were then fixed in 10\% neutral-buffered formalin for $48 \mathrm{~h}$, rinsed twice in running water, transferred to $70 \%$ ethanol, and processed into paraffin blocks. The processed tissues were stained with $\mathrm{H} \& \mathrm{E}$.

\section{Direct Immunofluorescence}

Direct immunofluorescence (DIF) of kidneys from MRL/ lpr mice was performed at $14 \mathrm{w}$. MRL/lpr mice were killed by dislocation. The mice were dissected on an ultraclean workbench, and the kidney from one side was quickly obtained. Then, the kidneys were frozen, and the deposition of immune complexes in the kidneys of MRL/lpr mice was observed with an Alexa Fluor 488-conjugated reagent under a microscope. The changes in immune complex deposition in the renal tissue from different groups were observed under a microscope.

\section{Extracting the Spleen and Obtaining Splenocytes}

The spleens were removed, weighed to evaluate splenomegaly, chopped with a sterile scalpel and crushed in a mortar with a sterile pestle using a fine mesh metal sieve in a Petri dish with RPMI 1640 medium. The cell suspension was transferred to a Falcon tube and allowed to stand for approximately $2 \mathrm{~min}$ for precipitation of larger tissue blocks. The supernatant was transferred to another Falcon tube and centrifuged (1200 rpm for $10 \mathrm{~min}$ at $4^{\circ} \mathrm{C}$ ). The pellet containing splenocytes was washed twice with RPMI 1640 medium and cryopreserved with dimethyl sulfoxide (DMSO) and fetal bovine serum (FBS).

\section{Flow Cytometry}

Lymphocytes were collected from splenocytes, and CD3+ $\mathrm{T}$ lymphocytes were collected with magnetic beads (Miltenyi Biotec). The samples were divided into the blank control, Con, Con $+0.5 \mu \mathrm{mol} 17-\mathrm{AAG}$, and Con +1 $\mu \mathrm{mol} 17-\mathrm{AAG}$ for observation. Then, the cell culture was incubated for $48 \mathrm{~h}$. The cell suspension was concentrated to $100 \mu \mathrm{L}$, and an anti-CD69 monoclonal antibody was added to each cell culture for $1 \mathrm{~h}$. The cells were washed with PBS, centrifuged and resuspended in a volume of 500 $\mu \mathrm{L}$. CD69+ T lymphocytes were counted using a FACS Calibur flow cytometer (BD) and a CD69 kit (Abcam). Automated analysis was performed using MULTISET software to calculate CD69+ T lymphocyte counts.

\section{Western Blotting}

Splenocytes were collected and washed twice with PBS, and protein was extracted with RIPA lysis buffer (Beyotime 
Biotechnology). The protein concentration was detected using a bicinchoninic acid (BCA) protein assay kit (Beyotime), and the proteins were separated electrophoretically via SDSPAGE, transferred to a PVDF membrane, and sealed with $5 \%$ bovine serum albumin (BSA, Sigma-Aldrich) for 1 h. The membranes were incubated overnight at $4^{\circ} \mathrm{C}$ with antibodies specific for HSP90 $\beta$, pAKTThr308, pGSK3 $\beta$ Ser9, AKT and GSK3 $\beta$ (Cell Signaling Technology, CST) (dilution ratios for Western blotting: $\beta$-ACTIN 1:5000, Hsp90 1:1000, AKT 1:1000, pAKTThr308 1:500, GSK3 $\beta$ 1:1000, and pGSK3ßSer9 1:500) and then incubated with anti-rabbit IgG as a secondary antibody (CST) for $1 \mathrm{~h}$. Differences in target protein expression were detected by the chemiluminescence method.

\section{Detection of the Proliferation of Splenic Lymphocytes with MTT}

The splenic lymphocytes of the experimental group and the control group were cultured $\left(10^{3}\right.$ cells were added to each well for culture). Three wells from each individual in each group were randomly selected every day. Dimethyl sulfoxide (DMSO) was used as the blank control. The growth of spleen lymphocytes was detected by the MTT method. The samples were detected for 7 consecutive days. A cellular growth curve was then generated.
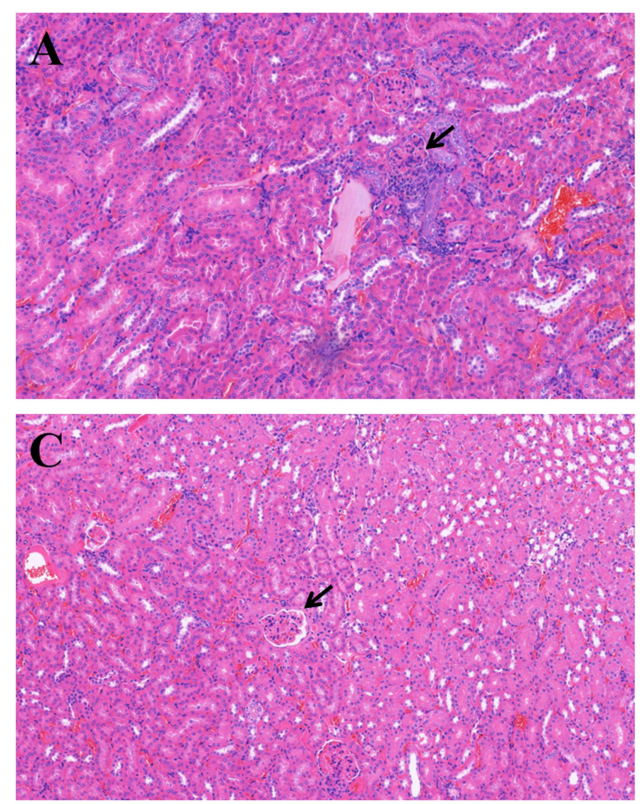

\section{Statistical Analysis}

SPSS 24.0 software was used for statistical analysis, and measurement data are expressed as mean \pm SD. A Student's $t$-test was performed to analyze the significance of changes in the mice. $p<0.05$ indicates a statistically significant difference.

\section{Results}

\section{Therapeutic Treatment with I7-AGG Ameliorates Renal Damage in MRL/lpr Mice}

HE staining was used to assess the severity of kidney inflammation in lupus mice. Compared to that in the control group, the degree of glomerular inflammatory infiltration in the experimental group was lower. In the control group, mild glomerulosclerosis, atrophy, partial vascular occlusion, obvious infiltration of interstitial lymphocytes between glomeruli and tubules, and fragmentation were observed (Figure 1). DIF results showed that there was stronger and more punctate fluorescence of immune complexes in the control group than in the experimental group, which suggested that the deposition of immune complexes in the experimental group was lower than that in the control group (Figure 2).

\section{Flow Cytometry}

After T lymphocyte culture for $1 \mathrm{~h}$, the expression of the early activated antigen CD69 in lymphocytes was detected
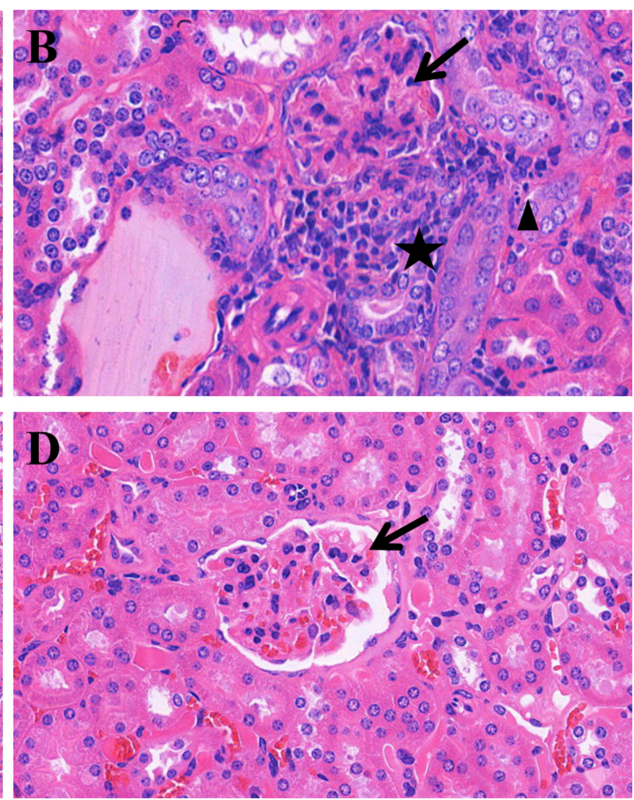

Figure I Representative H\&E staining for renal histopathology. In the control group, panel (A) (H\&E stain, I00x) and panel (B) (H\&E stain, 400x), glomerulosclerosis, atrophy, partial vascular occlusion (arrow), obvious infiltration of lymphocytes between glomeruli and tubules (pentagram), fragmentation of a necrotic nucleus (triangle) and proliferation of the basement membrane were observed. In the experimental group (17-AAG treated group), panel (C) (H\&E stain, I00x) and panel (D) (H\&E stain, 400×), the degree of glomerular inflammatory infiltration (arrow) was lower than that in the control groups. 

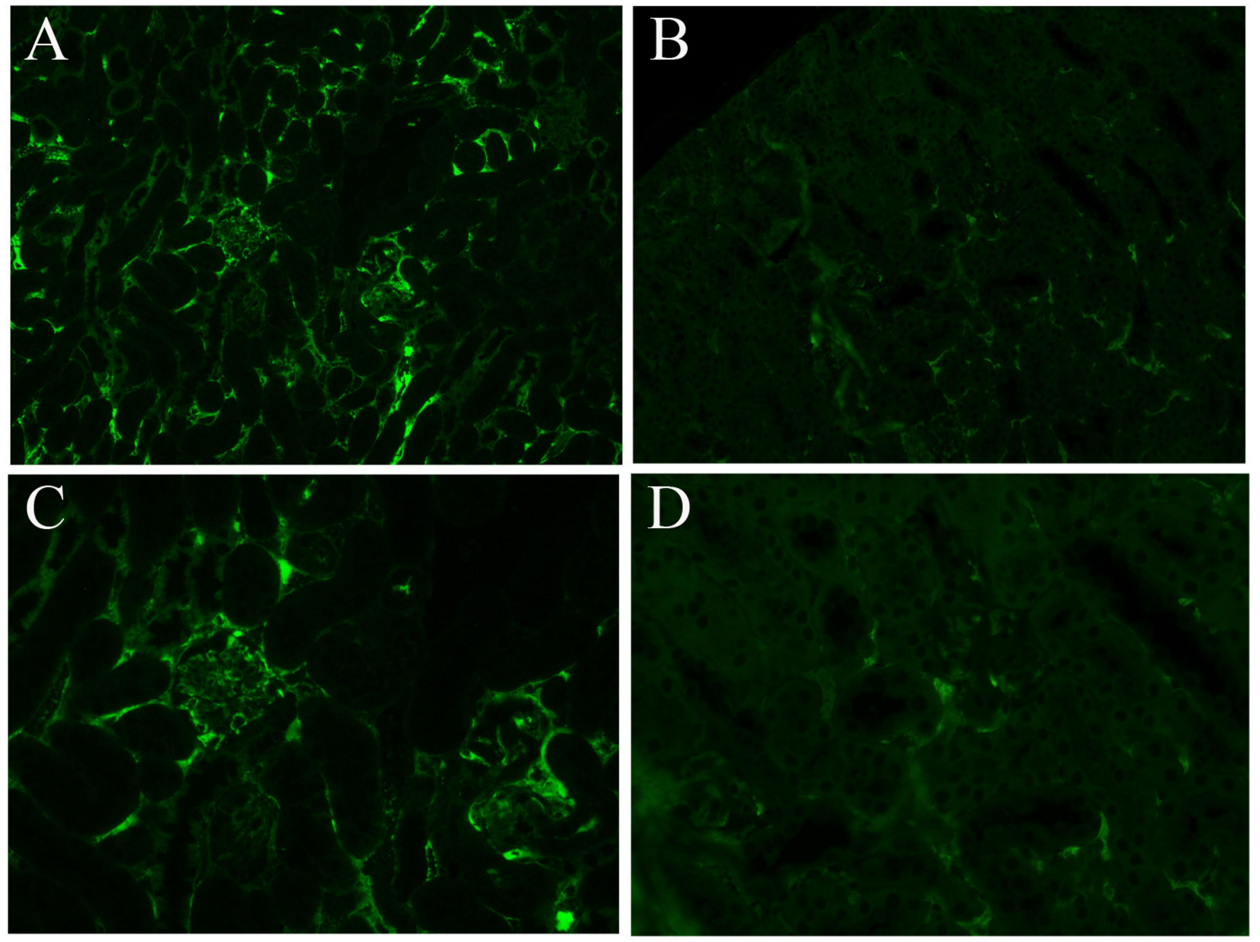

Figure 2 Analysis of renal immunofluorescence changes in the two groups. Panel (A) (100x) and panel (C) (400x) show the control group, panel (B) (I00x) and panel (D) $(400 \times)$ show the experimental group. The immunofluorescence of the experimental group was weaker than that of the control group, suggesting that the deposition of immune complexes was decreased.

by antibody staining combined with flow cytometry. The expression of CD69 in the control mice increased significantly after $6 \mathrm{~h}$ of ConA stimulation, and this expression decreased significantly after the addition of 17-AAG, which suggested that $17-\mathrm{AAG}$ could inhibit the activation and proliferation of $\mathrm{T}$ lymphocytes (Figure 3 ).

\section{Detection of the Expression of} PAKTThr308, pGSK3 $\beta$ Ser9, AKT and GSK3 $\beta$ in Splenic Lymphocytes from Lupus Mice

After treatment with 17-AAG, the expression levels, signaling protein activity and phosphorylation of both AKT and GSK $3 \beta$ were reduced in the experimental group; in addition, pAKTThr308 and pGSK3 $\beta$ Ser9 expression was inhibited, and AKT and GSK3 $\beta$ expression was not affected, suggesting that the phosphorylation of AKT and GSK3 $\beta$ was inhibited. 17-AAG can inhibit abnormal activation of the AKT/GSK3 $\beta$ signaling pathway in lymphocytes from mice with lupus (Figure 4).

\section{MTT Test}

For the MTT test without mitogen stimulation, mouse splenic lymphocytes were cultured in a 5\% carbon dioxide incubator at $37^{\circ} \mathrm{C}$ and observed for 7 consecutive days. The OD value of the control group was obviously higher than that of the experimental group, indicating that the proliferation of mouse splenic lymphocytes from the control group was obviously stronger than that of mouse splenic lymphocytes from the experimental group. Lymphocyte proliferation was inhibited by 17-AAG treatment in the experimental group (Figure 5).

\section{Discussion}

Heat shock proteins (HSPs) are a highly conserved family of proteins with important physiological functions. HSPs not only participate in protein transport and depolymerization and the protection of cells from environmental stress but also play an important role in autoimmunity and tumor immunity. Previous studies have shown that the expression of HSP90 and HSP90 antibodies is significantly increased in patients with SLE and is closely related to the degree of disease activity. However, HSP65 and HSP73 levels did not change in patients with SLE. Another study also found HSP90 antibody deposition in the glomeruli of patients with SLE. ${ }^{11}$ These results suggested that the high expression of HSP90 in patients with SLE played an important role in the disease. Therefore, HSP90 inhibitors may provide a new approach for the treatment of SLE. 

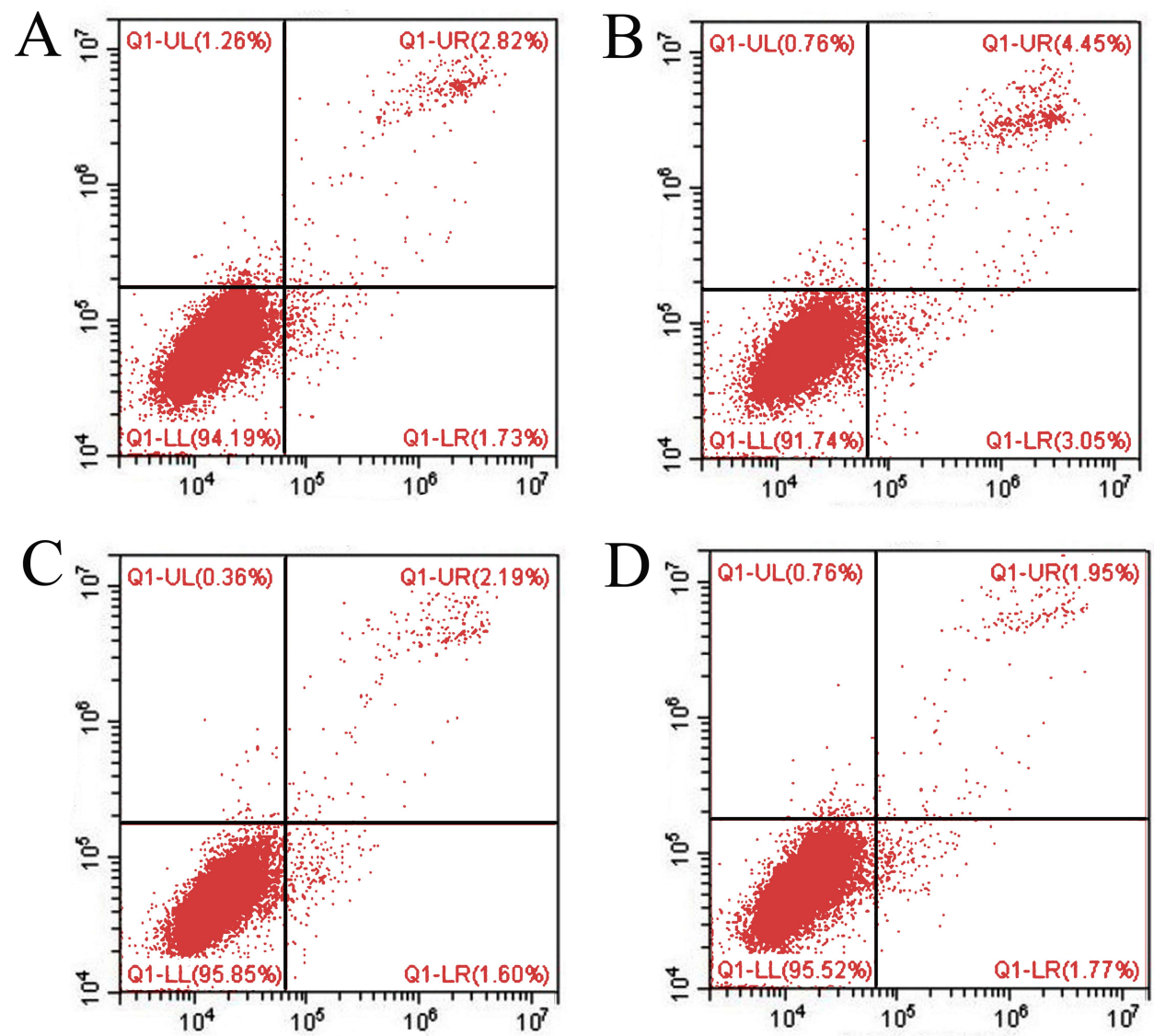

Figure 3 The expression of CD69+ lymphocytes was detected by flow cytometry. Group (A) had no intervention, group (B) was stimulated with ConA, group (C) was cultured with ConA+ $0.5 \mu \mathrm{mol}$ 17-AGG, and group (D) was cultured with ConA+ I $\mu$ mol 17-AGG. The expression of CD69+ lymphocytes decreased significantly after the addition of I7-AAG, which suggested that I7-AAG could inhibit the activation and proliferation of T lymphocytes.
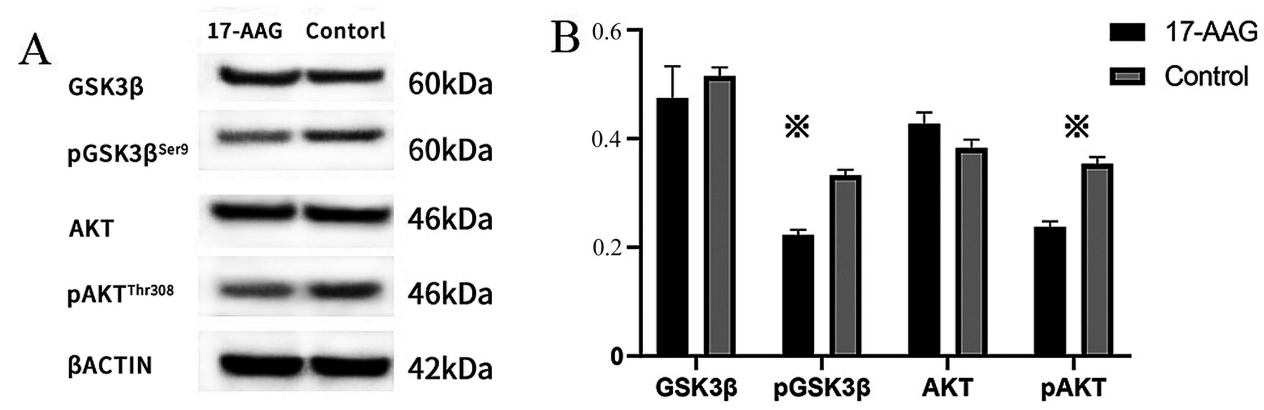

Figure 4 The expression of proteins in AKT/GSK3 3 signaling pathway. (A) Levels of signaling proteins were detected by Western blotting. (B) Compared to that in the control group, the phosphorylation of AKT and GSK3 $\beta$ in the experimental group was reduced, while AKT and GSK $3 \beta$ expression was not changed. Values represent the mean $\pm S D$. $* P<0.05$.

Recently, researchers have revealed the close relationship between HSP90 and tumors and reported that the inhibition of HSP90 can regulate the stability and activity of various substrate proteins to achieve anticancer effects. HSP90 client proteins not only affect tumor immunity but also participate in autoimmunity. Yun TJ showed that bone resorption, cartilage destruction and palmoplantar inflammation and swelling completely subsided or were significantly improved in rheumatoid arthritis model mice treated with EC144, a synthetic inhibitor of HSP90. ${ }^{12}$ Previous studies have reported the therapeutic benefit of targeting HSP90 in an autoimmune disease model of uveitis, 


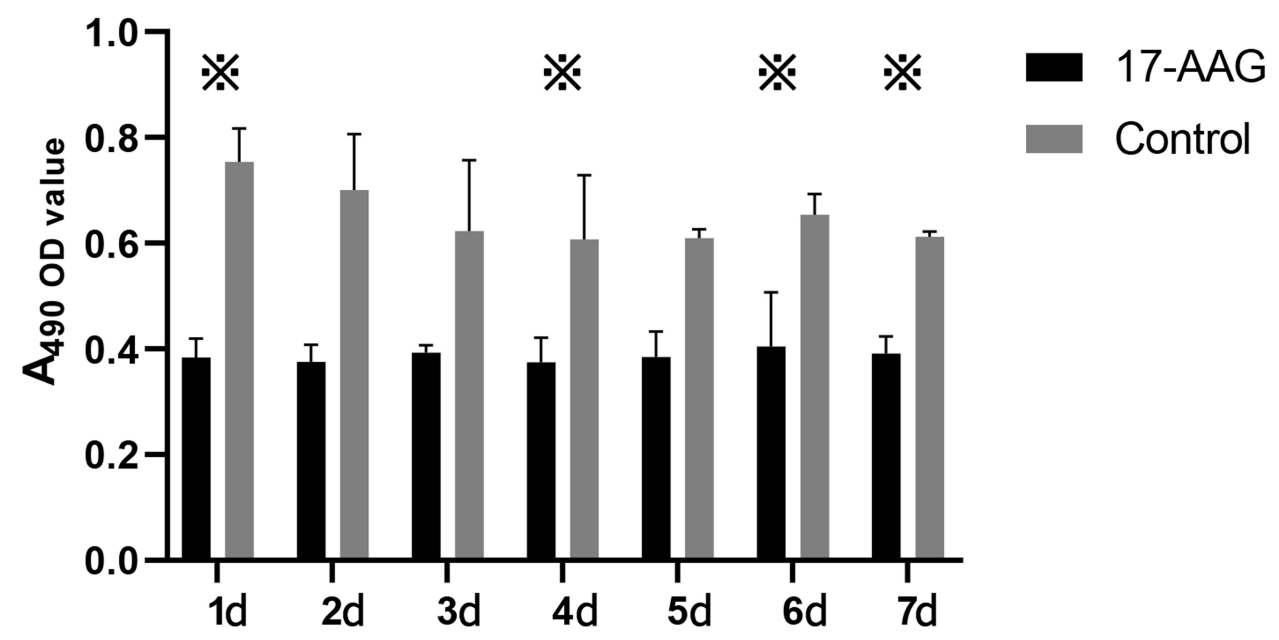

Figure 5 Comparison of the OD values of lymphocytes from MRL/lpr mice in the MTT assay. The changes in proliferation in the two groups of mice were recorded over one week. Values represent the mean \pm SD. ${ }^{*} p<0.05$.

experimental autoimmune encephalitis, and rat arthritis using both annamycin-derived inhibitors and a fully synthetic HSP90 inhibitor. ${ }^{12-14}$ However, only a few reports concerned HSP90 inhibitors in the treatment of SLE. In this report, we demonstrated that the HSP90 inhibitor 17AAG was an effective therapy in an inflammatory mouse model of lupus. ${ }^{15}$

The MRL/lpr lupus mouse is a publicly recognized model for the study of SLE. MRL/lpr mice are a model for the regulation of apoptosis caused by single-gene (Fas) mutations. 17-AAG was the first HSP90 inhibitor to enter clinical trials, and it has the characteristics of low toxicity and good inhibitory effects. In our study, we found that after treatment with 17-AAG in MRL/lpr lupus mice, the renal histopathology of the experimental group showed less inflammation and weaker immunofluorescence than observed in the control group. Our study confirmed that 17-AAG could effectively relieve lupus nephritis. Excessive activation of lymphocytes plays a key role in the pathogenesis of SLE. Activated autoreactive T cells in SLE stimulate the proliferation of autoreactive B cells. Resting T lymphocytes rarely express CD69, while activated $\mathrm{T}$ lymphocytes abundantly express this factor through transcription of encoded genes and the generation of large amounts of IL-2 and high-affinity IL-2R. IL-2and IL-2R-specific binding selectively supports amplification of $\mathrm{T}$ lymphocytes activated by antigen stimulation, so CD69 can be used as an early activation marker of T lymphocytes. ${ }^{16,17}$ Our study confirmed that under treatment with 17-AAG, the expression of CD69 in $\mathrm{T}$ lymphocytes of MRL/lpr mice was inhibited, which suggested that 17-AAG could inhibit the activation and proliferation of $\mathrm{T}$ lymphocytes.

Heat shock protein 90 is an ATP-dependent molecular chaperone that is required for the activation and stabilization of a wide variety of client proteins, and many of them are involved in important cellular pathways. HSP90 interacts with a variety of key signaling molecules to regulate growth, development, and death. Protein kinase B (AKT) is an important client protein of HSP90. Previous studies have found that the binding of HSP90 and AKT could stabilize activated AKT. Decomposition of the complex accelerates the dephosphorylation of AKT and reduces the activity of AKT protein kinase. ${ }^{18,19}$ AKT is an important mediator of lymphocyte proliferation and survival signaling downstream of PI3K. There are two phosphorylation sites, Ser473 and Thr308. Phosphorylated AKT is usually used as a marker of PI3K activation. Overactivation of AKT and phosphorylation of GSK3 $\beta$ can promote cell proliferation. AKT can regulate proteins related to the cell cycle and activate a signaling cascade. Its substrates include cell cycle factors such as GSK3 $\beta$. GSK3 $\beta$ can cause the phosphorylation of proteins such as cyclin D, which plays an important role in regulating cell differentiation and cellular responses. ${ }^{20} \mathrm{AKT}$ is the most important GSK3 $\beta$ kinase. AKT can inhibit its activity by phosphorylating GSK3 $\beta$. After intervention with the HSP90 inhibitor 17-AAG, the expression of the HSP90 protein in lymphocytes of MRL/lpr lupus mice was decreased. Furthermore, lymphocyte activation and the AKT/GSK3 $\beta$ signaling pathway were inhibited in the experimental group, and the levels of phosphorylated AKT and phosphorylated GSK3 $\beta$ were decreased. This 
study revealed that $17-\mathrm{AAG}$ could effectively inhibit the abnormal activation of lymphocytes in mice with lupus by downregulating the AKT/GSK3 $\beta$ signaling pathway, which may have potential for effective treatment of SLE.

\section{Conclusion}

AKT is a well-characterized target of PI3K. We downregulated activated AKT activity with 17-AAG and observed strong inhibition of AKT phosphorylation, a decrease in cell viability, and a reduction in the percentage of surviving cells, with a reduction in the activation and proliferation of $\mathrm{T}$ lymphocytes; this suggested that the possible mechanism underlying 17-AAG treatment of SLE was related to the AKT/ GSK3 $\beta$ signaling pathway. Our study revealed that pharmacological inhibition of the HSP90 chaperone pathway represents a potentially effective strategy for the treatment of SLE.

\section{Ethics Approval and Consent to Participate}

The present study was approved by the Ethics Committee of The First Affiliated Hospital of Chongqing Medical University (Chongqing, China). All experimental protocols were performed according to the Guidelines for the Care and Use of Laboratory Animals and were approved by the Institutional Ethics Committee of Chongqing Medical University, and conformed to the National Institutes of Health Guide for Care and Use of Laboratory Animals.

\section{Author Contributions}

All authors made a significant contribution to the work reported, whether that is in the conception, study design, execution, acquisition of data, analysis and interpretation, or in all these areas; took part in drafting, revising or critically reviewing the article; gave final approval of the version to be published; have agreed on the journal to which the article has been submitted; and agree to be accountable for all aspects of the work.

\section{Funding}

The present study was supported by grants from the National Natural Science Foundation of China (No.81602752) and Chongqing Research Program of Basic Research and Frontier Technology (No. cstc2015jcyjA10082).

\section{Disclosure}

The authors declare no conflicts of interest in this work.

\section{References}

1. Li J, Buchner J. Structure, function and regulation of the HSP90 machinery. Biomed J. 2013;36(3):106-117.

2. Prodromou C. Mechanisms of HSP90 regulation. Biochem J. 2016;473(16):2439-2452. doi:10.1042/BCJ20160005

3. Zininga T, Ramatsui L, Shonhai A. Heat shock proteins as immunomodulant. Molecules. 2018;23(11):2846. doi:10.3390/ molecules23112846

4. Tsan MF, Gao B. Heat shock protein and innate immunity. Cell Mol Immunol. 2004;1(4):274-279.

5. Wang Y, McAlpine SR. Heat-shock protein 90 inhibitors: will they ever succeed as chemotherapeutics? Future Med Chem. 2015;7 (2):87-90. doi:10.4155/fmc.14.154

6. Das JK, Xiong X, Ren X, Yang JM, Song J. Heat shock proteins in cancer immunotherapy. J Oncol. 2019;2019:3267207. doi:10.1155/ 2019/3267207

7. Saito K, Kukita K, Kutomi G, et al. Heat shock protein 90 associates with toll-like receptors $7 / 9$ and mediates self-nucleic acid recognition in SLE. Eur J Immunol. 2015;45(7):2028-2041. doi:10.1002/ eji.201445293

8. Shukla HD, Pitha PM. Role of HSP90 in systemic lupus erythematosus and its clinical relevance. Autoimmune Dis. 2012;2012:728605.

9. Kenderov A, Minkova V, Mihailova D, et al. Lupus-specific kidney deposits of HSP90 are associated with altered IgG idiotypic interactions of anti-HSP90 autoantibodies. Clin Exp Immunol. 2002;129 (1):169-176. doi:10.1046/j.1365-2249.2002.01887.x

10. Ripley BJ, Isenberg DA, Latchman DS. Elevated levels of the $90 \mathrm{kDa}$ heat shock protein (hsp90) in SLE correlate with levels of IL-6 and autoantibodies to hsp90. J Autoimmun. 2001;17(4):341-346. doi:10.1006/jaut.2001.0549

11. Dhillon VB, McCallum S, Norton P, et al. Differential heat shock protein overexpression and its clinical relevance in systemic lupus erythematosus. Ann Rheum Dis. 1993;52(6):436-442. doi:10.1136/ard.52.6.436

12. Yun TJ, Harning EK, Giza K, et al. EC144, a synthetic inhibitor of heat shock protein 90, blocks innate and adaptive immune responses in models of inflammation and autoimmunity. J Immunol. 2011;186 (1):563-575. doi:10.4049/jimmunol.1000222

13. Poulaki V, Iliaki E, Mitsiades N, et al. Inhibition of HSP90 attenuates inflammation in endotoxin-induced uveitis. FASEB J. 2007;21 (9):2113-2123. doi:10.1096/fj.06-7637com

14. Dello Russo C, Polak PE, Mercado PR, et al. The heat-shock protein 90 inhibitor 17-allylamino-17-demethoxygeldanamycin suppresses glial inflammatory responses and ameliorates experimental autoimmune encephalomyelitis. $J$ Neurochem. 2006;99(5):1351-1362. doi:10.1111/j.1471-4159.2006.04221.x

15. Liu Y, Ye J, Shin Ogawa L, et al. The HSP90 inhibitor ganetespib alleviates disease progression and augments intermittent cyclophosphamide therapy in the MRL/lpr mouse model of systemic lupus erythematosus. PLoS One. 2015;10(5):e0127361. doi:10.1371/journal.pone.0127361

16. Tomkowicz B, Walsh E, Cotty A, et al. TIM-3 suppresses anti-CD3/ CD28-induced TCR activation and IL-2 expression through the NFAT signaling pathway. PLoS One. 2015;10(10):e0140694. doi:10.1371/journal.pone.0140694

17. Cibrián D, Sánchez-Madrid F. CD69: from activation marker to metabolic gatekeeper. Eur J Immunol. 2017;47(6):946-953.

18. Liu K, Jin H, Guo Y, et al. CFTR interacts with HSP90 and regulates the phosphorylation of AKT and ERK1/2 in colorectal cancer cells. FEBS Open Bio. 2019;9(6):1119-1127. doi:10.1002/2211-5463.12641

19. Abeyrathna P, Su Y. The critical role of AKT in cardiovascular function. Vascul Pharmacol. 2015;74:38-48. doi:10.1016/j. vph.2015.05.008

20. Lin JX, Xie XS, Weng XF, et al. Overexpression of IC53d promotes the proliferation of gastric cancer cells by activating the AKT/ GSK3ß/cyclin D1 signaling pathway. Oncol Rep. 2019;41 (5):2739-2752. 


\section{Publish your work in this journal}

Drug Design, Development and Therapy is an international, peerreviewed open-access journal that spans the spectrum of drug design and development through to clinical applications. Clinical outcomes, patient safety, and programs for the development and effective, safe, and sustained use of medicines are a feature of the journal, which has also been accepted for indexing on PubMed Central. The manuscript management system is completely online and includes a very quick and fair peer-review system, which is all easy to use. Visit http://www. dovepress.com/testimonials.php to read real quotes from published authors. 